Archives de sciences sociales des religions

135 | juillet - septembre 2006

Réveils du soufisme en Afrique et en Asie

\title{
Introduction : le renouveau de l'islam soufi
}

Chanfi Ahmed

\section{OpenEdition}

Journals

Édition électronique

URL : http://journals.openedition.org/assr/3685

DOI : 10.4000/assr.3685

ISSN : $1777-5825$

Éditeur

Éditions de l'EHESS

Édition imprimée

Date de publication : 1 septembre 2006

Pagination : 9-15

ISBN : 2-7132-2093-9

ISSN : 0335-5985

Référence électronique

Chanfi Ahmed, «Introduction : le renouveau de l'islam soufi », Archives de sciences sociales des religions [En ligne], 135 | juillet - septembre 2006, mis en ligne le 16 octobre 2006, consulté le 21 septembre 2020. URL : http://journals.openedition.org/assr/3685; DOI : https://doi.org/10.4000/assr. 3685 


\section{Chanfi Ahmed}

\section{Introduction ${ }^{1}$ : le renouveau de l'islam soufi}

Depuis la période de la colonisation européenne des pays musulmans, beaucoup d'études ont été faites sur de multiples aspects des ordres mystiques musulmans (turuq, sing. tarîqa), ou confréries soufies : leurs pratiques populaires telles que les visites sur les tombes des saints, les danses extatiques; les complots et les rébellions, réels et supposés, qu'ils ont conduits contre l'État colonial; les périodes pendant lesquelles ils ont collaboré avec l'État et s'y sont adaptés tout en faisant continûment l'objet de surveillance de la part du pouvoir colonial ${ }^{2}$, etc. Avec la décolonisation de la fin des années 1950 et le début des années 1960, les élites qui dirigeaient alors les nouveaux États indépendants ont tout fait pour marginaliser les confréries au double motif qu'elles avaient des pratiques obscurantistes contraires aux idéaux de la sécularisation, du nationalisme, du socialisme, du progrès, de la modernité, etc., ensuite parce qu'elles avaient collaboré avec les colonisateurs européens. À la fin des années 1970, et surtout depuis les années 1980, on assiste à la montée en puissance des mouvements de l'islam politique, lesquels, il est vrai, attaquent, en premier lieu, les pouvoirs politiques en place auxquels ils reprochent d'avoir échoué dans leur mission de réalisateurs du progrès, et cela parce qu'ils ne gouvernent pas selon la loi d'Allah (la sharî́a) et les «vraies » valeurs de l'islam. Ces mouvements s'appuient, en grande partie, sur l'idéologie politique des Frères musulmans (Ikhwân al-muslimûn) et sur la doctrine salafiyya et wahbâbiyya en ce qui concerne la croyance et la pratique religieuses. Or, cette doctrine entend, justement, promouvoir un islam monolithique "purifié » des pratiques culturelles locales qu'elle considère soit comme des bida (sing. bid'a : innovation blamâble), des shirk ${ }^{3}$ (litt. associationnisme), soit

1. Je remercie Marc Gaborieau d'avoir lu et commenté cette introduction. Une grande partie de ses remarques y sont intégrées.

2. Sur ce thème de l'adaptation et de la surveillance dont les turuq faisaient l'objet, voir surtout David Robinson, "The Murids: Surveillance and Collaboration ", Journal of African History, 40, 1999, p. 193-213 ; David Robinson, Paths of Accommodation. Muslim Societies and French Colonial Authorities in Senegal and Mauritania, 1880-1920, Athens, Ohio University Press, Oxford, James Currey, 2000.

3. C'est " associer autre chose " (d'où le terme associationnisme) à la prière, à l'invocation et à l'adoration d'Allah. C'est croire que quelque chose ou quelqu'un d'autre que le Prophète Muhammad peut, avec l'agrément d'Allah, intercéder entre le croyant et Allah dans sa volonté toute-puissante. 
comme des khurâfât (mystifications, superstitions). De plus, comme les élites qui dirigeaient les premiers États indépendants, les leaders islamistes reprochent aux turuq d'avoir collaboré avec les puissances coloniales. La marginalisation des turuq par ces trois pouvoirs (le pouvoir colonial, celui des premières élites de l'État indépendant et celui des partisans de l'islam politique) a pu conduire spécialistes et non spécialistes à parler de la fin des turuq. Et pourtant il n'en est rien. Non seulement les confréries soufies ne disparaissent pas, mais elles se revigorent en s'adaptant au monde moderne aussi bien sur le plan doctrinal que sur celui de l'organisation.

Le regain d'intérêt que suscite l'islam aujourd'hui dans les recherches en sciences sociales et historiques porte surtout sur l'islam politique et sa revendication de pratiques cultuelles "pures » qui feraient abstraction des influences des cultures locales, un islam global qu'on pourrait pratiquer de la même façon aussi bien dans les pays du Sud que dans ceux du Nord. C'est l'islam que les activistes appellent al-sahwa al-islâmiyya (réveil islamique) et que la littérature médiatique rend par différents « ismes » : fondamentalisme, islamisme, radicalisme, intégrisme, etc. Le mouvement que l'on nomme couramment le " néo-soufisme " ${ }^{4}$ appelle - curieusement comme ses adversaires wahbâbiyya et salafiyya et pour parer aux critiques de ces derniers - à purifier le soufisme des pratiques " non orthodoxes » et contraires à l'« idéal soufi ", et illustre à titre d'exemple, cet effort d'adaptation des turuq sur le plan doctrinal. Les turuq, et les mouvements prosélytes comme le tabligh, sont non seulement globaux et transcontinentaux depuis longtemps, mais ils renouvellent leur organisation en réseaux translocaux pour faire face au défi que représentent pour eux les activités des mouvements de la sahwa al-islâmiyya, les crises de toutes sortes que connaissent les sociétés islamiques aujourd'hui et les exigences du monde moderne. Peut-on, dans ce cas, parler d'un "réveil soufi » (sahwa sûfiyya) ? Et si oui, comment se manifeste-t-il concrètement ? C'est la question principale à laquelle les différentes contributions de ce recueil essaient de répondre, chacune selon ses terrains d'approche et sa perspective. Une confrérie comme la Shâdhiliyya Yashrutiyya en Afrique de l'Est se revivifie et se perpétue à travers les cérémonies de commémoration et les ziyâra (visites, pèlerinages) que les disciples accomplissent sur les tombeaux des fondateurs de la tarîqa. Les ziyâra créent dans le même temps une culture de mémoire, et des réseaux translocaux et transrégionaux. D'un autre côté, le mouvement de mission islamique "transcontinental » qu'est la Tablîghî Jamâ‘at semble être aux antipodes du soufisme. Et pourtant, il recèle dans son sein un substrat soufi qui sert en quelque sorte de levier à ses militants dans leur travail de mission.

Les contributions publiées ici reflètent à des degrés divers l'entrelacement des deux thèmes de la translocalité et du prosélytisme, auxquels se surajoute le thème

4. Sur le débat autour de la notion du "néo-soufisme ", voir surtout R. Sean O'FAHEY, Bernd RadtKe, "Neo-Sufism Reconsidered ", Der Islam, IXX, 1993, p. 52 ff. 
dominant de la réforme ${ }^{5}$. Le premier thème, la translocalité, mis en évidence par le premier article de ce recueil, est abordé dans son aspect dynamique, que ce soit dans l'histoire des groupes soufis étudiés, dans leurs réseaux et dans leurs rituels principaux. Par contre, dans les trois articles placés au centre, c'est le thème du prosélytisme qui vient au premier plan. Enfin, le thème abordé par presque tous les textes présentés est celui de la réforme des pratiques soufies, qui est particulièrement en évidence dans les trois contributions qui viennent en fin de ce recueil.

L'organisation en réseaux est consubstantielle aux turuq depuis leur apparition (entre le XII et le XIV ${ }^{\mathrm{e}}$ siècle), mais elle s'était longtemps développée au Sud uniquement. C'est seulement à partir $\mathrm{du} \mathrm{Xx}^{\mathrm{e}}$ siècle, à la faveur surtout du développement des moyens de transports et de la révolution des techniques de communication, que les turuq ont pris pied au Nord (Europe, Amérique) et s'y sont développées. La contribution de Roman Loimeier, centrée sur la translocalité, montre bien comment l'organisation traditionnelle des ordres soufis en réseaux s'adapte facilement au contexte contemporain de la mondialisation. L'étude des liens translocaux de la confrérie Qâdiriyya entre Zanzibar (Afrique de l'Est), le Nigeria (Afrique de l'Ouest) et le centre de la tarîqa à Baghdad en Irak, montre comment ceux-ci fonctionnent sur trois niveaux qui s'interpénètrent : celui des guides ('ulamâ) de la tarîqa de ces trois localités ou régions ; celui de l'enseignement de la tarîqa et de l'enseignement islamique en général ; et celui des rituels et des polémiques que se livrent à leur sujet les réformistes et les traditionalistes. En ce qui concerne le premier niveau, l'auteur présente les liens qui se sont tissés entre 'Umar Abdallah de Zanzibar, Nasiru Kaba du Nigeria et Sheikh Saif ad-Dîn b. 'Abd al-Qâdir al-Jilânî, descendant de 'Abd al-Qâdir al-Jilânî (m. 1166), le fondateur de la tarîqa à Baghdad. Ces liens ont été exploités par les personnalités concernées non seulement pour maximiser leur prestige, mais aussi pour faire avancer l'enseignement islamique dans la tradition de leur tarîqa en concurrençant dans ce domaine les réformistes aussi bien que les autres turuq. Roman Loimeier montre d'une manière éclatante comment les deux thèmes de la réforme et de la translocalité se cristallisent dans la polémique autour de l'usage contesté de la musique des tambourins ( $d u f u$, bandîr) dans le rituel de $d h i k r$ à la fois à Zanzibar et au Nigeria. On y apprend, par exemple, que l'usage de la musique des tambourins dans le $d h i k r$ de la Qâdiriyya à Zanzibar et au Nigeria n'était pas du tout une marque de "contextualisation » dans le milieu africain d'une

5. Les articles réunis ici sont principalement issus d'un colloque tenu à Berlin les 4-5 avril 2003 dans le Centre de l'Orient Moderne (ZMO : Zentrum Moderner Orient, Centre For Modern Oriental Studies). Ce centre qui est dirigé par Mme la Prof. Ulrike Freitag, historienne du Proche-Orient, est le seul centre de recherche en Allemagne à avoir une approche interdisciplinaire et une perspective d'histoire comparée sur le Proche-Orient, l'Afrique, l'Asie du Sud et du Sud-Est. Les interactions entre les sociétés musulmanes ainsi que les relations qu'elles entretiennent avec les régions voisines non musulmanes sont au cœur de ses préoccupations de recherche. Fondé en 1996, le ZMO est un centre extra-universitaire de recherche en sciences humaines et sociales. 
tarîqa venue de l'Orient arabe, mais que cet usage venait du centre même de la Qâdiriyya à Baghdad.

Les trois articles suivants traitent en premier lieu du prosélytisme à travers deux mouvements missionnaires d'origine indienne ${ }^{6}$. Les deux premiers de ces articles (Dietrich Reetz et Marc Gaborieau), se répondent en revenant sur une question depuis longtemps posée : les rapports au soufisme et au réformisme dans un même et unique mouvement transnational, la Tablîghî Jamâ'at créée en 1927. Le troisième article (Denise Brégand) ouvre sur un autre mouvement prosélyte qui est en fait plus ancien, puisqu'il remonte à la fin du XIX ${ }^{e}$ siècle, celui de la Ahmadiyya (sans doute le modèle inavoué de la Tablîghî Jamâ‘at).

Dietrich Reetz présente les Tablighî de quatre points de vue : les origines du mouvement, la manière dont les Tablighî mènent leur propagande, leur doctrine, et leurs relations avec les autres mouvements islamiques au Pakistan et en Inde. Ce mouvement réformiste, qui a intégré dans son sein l'héritage spirituel soufi, a été fondé par Muhammad Ilyâs en 1927 dans la région de Mewât aux alentours de Delhi au nord de l'Inde. Contrairement au mouvement réformiste qui a émergé dans le monde arabe au début du XIX siècle, d'abord en réaction contre le colonialisme européen, la Tablị̂̂i Jamâ'at est un mouvement réformiste endogène, né d'une situation locale créée par la propagande de conversion que menaient à l'époque les prêcheurs hindous au sein des tribus converties à l'islam. Dès les origines du mouvement, l'objectif était tout d'abord de reconvertir le converti, en d'autres termes de fortifier la foi du converti, et de provoquer de nouvelles conversions. Cet objectif a conduit les Tablîghî à user des mêmes armes que leurs protagonistes hindous en prêchant intensément. Mieux, ils ont perfectionné les techniques de prédication en imitant les prêcheurs itinérants des différents groupes protestants qui sillonnaient la région à l'époque. C'est d'eux, semble-t-il, qu'ils ont pris et appris leurs prêches de porte à porte, ou gâsht (patrouille: terme persan que les Tablîghî emploient en Inde et que les Tablîghî maghrébins en Europe nomment khurûj, litt. : sortie) pour inviter les musulmans et les nouveaux convertis à la mosquée de la localité afin de leur prêcher la bonne parole.

D. Reetz, après avoir également souligné l'aspect soufi de l'organisation, le questionne en cherchant à savoir comment et jusqu'à quel point les Tablîghî s'inspirent du soufisme dans la construction de leurs autorités et guides religieux, dans leurs formes d'organisation, dans la prise de décision et dans les interactions sociales au sein du mouvement ? Enfin, comment mettent-ils à contribution la spiritualité soufie pour en mobiliser les membres ? Si, d'après D. Reetz, le soufisme est une partie consubstantielle de la Tablîgî Jamâ‘at, il est inégalement réparti entre l'Inde et le Pakistan : dans le premier pays, l'aspect soufi du mouvement est resté très fort; dans le second, il se fait de moins en moins visible.

6. Sur ces mouvements, voir Catherine Clémentin-OjHa, Marc GaborieAu, « La montée du prosélytisme dans le sous-continent indien ", Archives de sciences sociales des religions, $\mathrm{n}^{\circ} 87$, juillet-septembre 1994. 
Marc Gaborieau conteste pour sa part le caractère soufi de la Tablîgî Jamâ'at, en rappelant d'abord la généalogie de ce vieux débat des études concernant ce mouvement. Deux traditions de recherche s'affrontent : la première, représentée par Muhammad Anwârul Haq, auteur de la première thèse sur les Tablîghî, soutient que la Tablîgî Jamâ'at est une organisation soufie, alors que la seconde, représentée par Khalid Masud, affirme qu'il s'agit tout simplement d'un mouvement missionnaire réformiste. M. Gaborieau - en phase avec D. Reetz - rejette la prétendue position apolitique des Tablîghî, mais il maintient néanmoins que le fait que les Tablîghî soient préoccupés de conquérir toujours plus d'espace par leur prédication est à rapprocher, particulièrement pour ce qui concerne leur organisation hiérarchique et presque militaire sous la direction d'un amîr, avec des patrouilles (gâsht ou khurûj), du mouvement du jihâd armé médiéval. Selon l'auteur le prétendu caractère soufi du mouvement, l'habit apolitique dont celui-ci se revêt, la soit-disant non-implication du mouvement dans les conflits interreligieux, et autres moyens auxquels il recourt pour se donner une image politically correct, tout cela relève d'une stratégie de légitimation qui masque le passé historique du mouvement.

Dans le troisième article, plus centré sur le prosélytisme, Denise Brégand revient sur le mouvement Ahmadiyya, fondé en Inde par Mirzâ Ghulâm Ahmad (c. 1838-1898), qui fut la première grande organisation missionnaire dans le monde musulman moderne, et qui inaugure, au niveau du sous-continent indien, ce que l'on a appelé « la montée du prosélytisme " ${ }^{7}$. La fondation de ce mouvement illustre la concurrence des musulmans et des hindous dans une lutte d'influence qui prend appui sur le modèle fourni par les missionnaires chrétiens, dont les uns et les autres avaient eu à souffrir. Les spéculations du fondateur avaient leurs racines dans le soufisme, et il se voyait comme un réformateur. Les efforts de ses successeurs ont visé avant tout à répandre l'islam dans le monde entier - véritable globalisation avant la lettre. Le présent article montre comment cette stratégie d'expansion est mise en œuvre aujourd'hui au Bénin, et comment elle s'adapte au contexte africain, selon que l'islam y est minoritaire ou majoritaire.

Dans les débats sur les Tablîghî, comme dans tous les autres articles, notamment ceux de Michael Laffan et de Samuli Schielke, un enjeu essentiel demeure en filigrane : celui de la réforme du soufisme. Michael Laffan rend compte du débat sur l'impératif de la réforme de l'islam en Indonésie en général, et du soufisme en particulier, face à l'idéologie de la globalisation. Il le fait à travers l'étude d'une revue mensuelle sur le soufisme qui a paru en Indonésie de 2000 à 2002; une vie assez longue dans le contexte de ce pays, d'après $M$. Laffan, pour une revue d'opinion. Plusieurs thèmes ont été traités dans cette revue, mais deux grandes tendances paraissent dominer toutes les livraisons. La première est constituée d'une série d'études sur les "pères » fondateurs du soufisme en islam en général (Ghazzâlî, Hallâj, Rûmî, Abd al-Qâdir al-Jilânî, etc.), les grands soufis

7. Catherine Clémentin-Ojha, Marc Gaborieau, op. cit. 
fondateurs de turuq en Indonésie (Ahmad Khatib Sambas, Ismail Minangkabau, etc.,) et aussi d'informations sur les orientalistes, comme Massignon, qui ont écrit sur le soufisme. La seconde tendance est formée d'articles et d'interviews portant sur le soufisme face à de grands thèmes, tels que la globalisation, l'hégémonie de la culture occidentale, la modernité. Il faut se rappeler que la revue paraissait dans une période pendant laquelle le pays connaissait un regain de propagande islamique dans différentes versions et une intensification des activités politiques à la suite de l'ouverture libérale qui a suivi la chute du régime de Suharto et l'arrivée d'Abdurrahman Wahid au pouvoir. Mais c'est en même temps la période qui a suivi la crise financière de 1997-1998 qui a durement frappé le pays. C'est dans cette situation d'ouverture politique et de crise économique que la revue paraissait. Et c'est à juste titre que $\mathrm{M}$. Laffan pose la question de savoir quel genre de remède la revue propose pour guérir ce grand mal.

Dans sa contribution, S. Schielke analyse les cérémonies populaires soufies des mawlid - ou fêtes anniversaires des saints - en Égypte aujourd'hui, et les discours réformistes et rationalistes visant à les «moderniser ", à les "réformer ", et à les purifier pour en faire, dit-on, des "pratiques islamiques authentiques ". Depuis l'apparition des mawlid il y a un millénaire, à l'époque des Fatimides, puis des Mamluks, les oulémas réformistes ont toujours critiqué leurs aspects populaires et festifs considérés par eux comme des bid'a (innovations blâmables) et contraires à $\mathrm{l}^{\prime}$ " islam authentique ». Aux XIX et $\mathrm{XX}^{\mathrm{e}}$ siècles, les modernistes, rejoignant les réformistes, les stigmatisent aussi comme des pratiques obscurantistes, irrationnelles et contraires au progrès et à la modernité. Ils dénonçaient (comme aujourd'hui) l'atmosphère festive, carnavalesque, des $d h i k r$ collectifs, avec leur lot de musiques et danses extatiques, le commerce informel, les pèlerinages sur les tombes des saints, la non séparation entre les femmes et les hommes, ainsi que d'autres aspects du mawlid qui dénotent à leurs yeux l'ignorance, la transgression des normes islamiques, le manque de rationalité et le désordre.

Pour contrecarrer les attaques des détracteurs du soufisme, l'État, les représentants officiels de l'islam - le recteur de l'université al-Azhar, le Supreme Council of Sufi Orders (al-Majlis al-al'â li-t-turuq as-sûfiyya) et les représentants du soufisme réformiste - ont engagé une série de mesures tendant à réformer et à moderniser les mawlid; ce qui se traduit par la tentative (réussie en grande partie) d'imposer du nizâm (à la fois ordre, discipline et organisation) dans le mawlid. L'espace dans lequel il est autorisé à s'accomplir est soumis partout à des frontières et des barrières (matérielles et/ou idéelles) séparant ce qui relève du mawlid authentique et ce qui en serait une déviation, même si le mawlid « inauthentique " ou " déviant " persiste toujours aux marges du premier, avec ses foules bigarrées, ses transgressions, son aspect festif et carnavalesque. S. Schielke souligne que les représentants de l'État et les soufis réformistes acceptent malgré eux cet aspect essentiel et consubstantiel du mawlid: en témoigne, ce que disent certains parmi eux : "C'est la foule qui fait le mawlid» (il-mûlid fî zahmituh). Après tout, le permis 
et le défendu, la norme religieuse et sa transgression, sont les deux faces d'une même médaille : chacune a besoin de l'autre pour exister.

A. Popovic aborde les deux thèmes de la translocalité et de la réforme à partir d'une périodisation historique qu'il applique à l'évolution du soufisme dans les pays balkaniques (Bosnie-Herzégovine, Kosovo, Macédoine et Albanie). Il montre que dès les premières conquêtes ottomanes dans la péninsule, aux XIV $\mathrm{XV}^{\mathrm{e}}$ siècles, des turuq comme la Bektâshiyya, la Naqshbandiyya se sont implantées. Elles furent suivies entre le XVI et le XVIII ${ }^{e}$ par d'autres comme la Qâdiriyya, la Rifấiyya. Et enfin au XIX ${ }^{e}$ siècle une troisième sorte de turuq comme la Melâmiyya-Nûriyya a fait son apparition. Dans chacune de ces périodes, les autorités ottomanes instrumentalisaient massivement ces turuq selon leurs besoins politiques du moment, notamment dans la colonisation des territoires et l'encadrement de l'armée. Les turuq se sont forgées, d'une période à l'autre, une habitude d'adaptation qui allait leur permettre de survivre dans les moments difficiles notamment après l'avènement des États-nations, puis sous les régimes communistes. La fin de la guerre froide a ouvert une nouvelle période du soufisme dans les Balkans. On y observe, en effet, un renouveau des anciens réseaux translocaux des turuq et l'apparition de nouveaux réseaux venant de Turquie, d'Iran et d'autres pays musulmans, voire même des différentes diasporas balkaniques en Europe et aux États-Unis d'Amérique. En même temps sont apparues sur la scène de l'islam balkanique différentes "missions islamiques ", de charité et d'enseignement islamique, dont la plupart sont contre les pratiques des turuq.

Les quelques contributions présentées ici prouvent, si besoin est, la vitalité de l'islam soufi qui s'exprime sous différents aspects. D'abord dans ses structures classiques que sont les turuq, ensuite dans de nouvelles institutions telles que les revues, et enfin, d'une manière voilée, dans des organisations missionnaires islamiques qui pourtant ne se réclament pas explicitement du soufisme. Les réformes engagées sont à la fois doctrinales et organisationnelles. Confronté à la poussée des mouvements islamistes qui revendiquent aussi leurs tendances réformistes et modernistes, le soufisme est forcé de relever le défi. Il ne faudrait pas voir cependant les réformes du soufisme et son action prosélyte comme une simple réaction aux mouvements islamistes. Il s'agit aussi et surtout de la marque d'une tradition soufie de survie et d'adaptation au monde qui change. En effet, ni les tentatives des pouvoirs politiques établis pour les marginaliser ou les instrumentaliser, ni les attaques dont elles ont toujours fait l'objet de la part des réformistes de la Salafiyya et autres islamistes n'ont rien entamé de leur dynamisme. 
